\title{
On the edge turbulence effects on the density profile reconstruction using O-mode reflectometers
}

\author{
E. Ricardo ", a, F. da Silva ${ }^{\text {a }}$, S. Heuraux ${ }^{\mathrm{b}}$, A. Silva ${ }^{\mathrm{a}}$ \\ ${ }^{a}$ Instituto de Plasmas e Fusão Nuclear, Instituto Superior Técnico, Av. Rovisco Pais, no. 1, Lisbon, Portugal \\ ${ }^{\mathrm{b}}$ Institut Jean Lamour UMR 7198 CNRS-Universite de Lorraine, ARTEM BP 50840, Nancy F-54011, France
}

\begin{abstract}
A B S T R A C T
The effect of turbulence in the position measurements of the DEMO Plasma position reflectometer is evaluated at the high field side equatorial plane location using REFMULF, a 2D finite-difference time-domain full-wave code for reflectometry simulations. Different levels of turbulence (1-10\%) of a Kolmogorov-like k-spectrum are analysed statistically with random generated density fluctuations. The study shows that for turbulence amplitudes in the range 1-7\% most of the measurements are not affected significantly and the reflectometer can provide the position measurements within the DEMO requirements. For higher levels of turbulence, the mean error becomes negative and its absolute value and the standard deviation increases for values in the order of 0.5 $\mathrm{cm}$. One of the reasons for this behavior is the deviation of the effective cut-off position. In these cases, part of the measurements are out of the requirements, and a study must be done to understand if the reconstruction of the separatrix stills possible for the order of error of the failed measurements $(1-2 \mathrm{~cm})$. Other possibility is to apply a correction based on the mean position value as function of the turbulence amplitude to the position measurements. This could be applied dynamically during the plasma pulse.
\end{abstract}

\section{Introduction}

Unlike the experimental machines, DEMO will only have the necessary diagnostics to provide the plasma state with the necessary accuracy for machine protection and plasma control [1]. A fundamental measurement for the control system is the position and shape of the last closed magnetic surface, initially measured with magnetic diagnostics in the current machines. One of the major issues of their implementation in DEMO is the large integration drifts that can occur during the long pulses due to the high levels of radiation, leading to a wrong plasma position estimation [1]. The first candidate to substitute the magnetic diagnostics in DEMO is Microwave reflectometry, fulfilling the needs/requirements to the operation [1,21]. This diagnostic is capable of measuring the electron density profile and its time evolution by sweeping the frequency of the probing beam in a very short time scale [15]. The density is directly linked to the magnetic flux surfaces and these measurements give access to the magnetic configuration, providing its local radial position. The use of O-mode reflectometry to measure the plasma position has been experimentally validated in real-time on ASDEX-Upgrade [20] and continues to be an active research topic.
The DEMO plasma position reflectometer (DEMO PPR) is a system of multi-reflectometers distributed poloidaly along the wall that will provide the measurements of different separatrix positions used to reconstruct its shape. The design and the optimization of the DEMO PPR is a complex task and requires the simulation of different poloidal views, emitting angles, antenna assemblies and plasma configurations. It must be optimized for the baseline operation scenario and must be stable under the deviations to its equilibrium that may occur during the machine operation. Many different phenomena as the plasma displacements, turbulence and MHD activity occur during the pulses and can affect the reflectometry measurements. Up to now, the DEMO PPR is just a concept in development stage and many questions need to be investigated before reaching its final design. A recent study tested 100 different positions around the wall using the 2015 baseline plasma [18]. The probing wave was aligned perpendicularly with the separatrix to minimize the effect of the plasma curvature. All the measurements with the exception of the divertor region higher curvature) are within the requirements for the plasma positioning system.

One fundamental question of the PPR performance is its reliability due to the effect of the turbulence in the measurements. It is experimentally know that density fluctuations influence the round-trip time

Keywords:

Microwave reflectometry

Plasma turbulence

Reflectometer

DEMO PPR 
delay of the probing wave and deflect part of its energy, resulting in the wrong position measurement and, in some cases, signal lost [26]. One of the fundamental mechanisms that affects the phase of the probing wave is the Bragg scattering. This corresponds to the plasma-wave interactions satisfying the Bragg rule, $\mathbf{k}_{f}=-2 \mathbf{k}\left(r_{B}\right)$, where $\mathbf{k}_{f}$ is the fluctuations wavenumber, $\mathbf{k}$ is the local wavenumber of the incident wave and $r_{B}$ is the Bragg position, where the condition is full-filled [5]. When a 1D model is assumed, this rule can be only satisfied in the range $2 k_{A}<k_{f}<2 k_{0}$, where $k_{A}$ is the Airy wavenumber $k_{A}=0.63\left(k_{0}^{2} L_{n}^{-1}\right)^{1 / 3}$ and $L_{n}$ the density gradient scale length. A variety of mechanisms are described analytically in the literature, including expressions to the phase shift obtained with the Born approximation and the non-linear regime $[7,17]$. Higher absolute changes in the phase of the probing wave is generally caused by higher amplitude fluctuations. Their amplitude and structure play an important role in the way how the energy is scattered to different directions.

Despite the advances in the reflectometry theory, the interaction between the electromagnetic field and the turbulent plasma is only partially described. The theoretical $1 \mathrm{D}$ and $2 \mathrm{D}$ models predict the behavior of some observed phenomena, typically for specific plasmas and beam shapes [13]. For this reason, the numerical simulations are essential to predict the reflectometry measurements encountered in realistic cases beyond the validity domain of the existing theories. With the $2 \mathrm{D}$ simulations, the reliability of the reflectometer can be estimated by the expected turbulence parameters. From the point of view of numerical simulations, testing the effect of turbulence in the measurements involves making a statistical study of the measurement error and of the received power for different values of the turbulence parameters, such as the amplitude of the fluctuations [12]. In this work we analyse the effect of the turbulence amplitude in the position measurement performance at the DEMO HFS equatorial plane location using REFMULF, a 2D finite-difference time-domain (FDTD) code for reflectometry simulations $[22,23]$. The plasma is described by the cold-plasma approximation and the surrounding metallic structure by a perfect conductor. The turbulence is modelated by a Kolmogorov-like k-spectrum [see Eq. (5)] and taking into account a $\rho_{S}$ scaling compatible with the order of the expected DEMO parameters [11]. Four hundred different plasmas with random spectral phase configurations are simulated for each turbulence amplitude, giving a good compromise between the computational time and the statistic error.

\section{Modeling and simulation results}

\subsection{The position error}

For a monotonic density profile, the cut-off position can be directly calculated using the Abel inversion [10,15],

$r_{c}(F)=r_{0}+\frac{c}{2 \pi^{2}} \int_{0}^{F} \frac{\mathrm{d} \phi}{\mathrm{d} f} \frac{1}{\sqrt{F^{2}-f^{2}}} d f$

where $r_{c}$ is the cut-off position, $\phi$ is the phase change of the wave due to the propagation in the plasma and $F$ is the probing frequency. For the $\mathrm{O}-$ mode, the cut-off density is

$n_{c}(F)=\alpha^{-1} F^{2}=\frac{1}{80.6164} F^{2}\left[m^{-3} s^{2}\right]$

where $\alpha=4 \pi^{2}\left(\varepsilon_{0} m_{e} / e^{2}\right)$. Knowing the phase derivative from 0 to $F$, or equivalently the round-trip time delay, the density profile $\left[r_{c}(F), n_{c}(F)\right]$ can be reconstructed. An initialization method is usually required due to the initial frequency is different from zero, where a shape for the phase derivative in the range $0-F_{i}$ is assumed. In a real reflectometer the complex form of the plasma-wave interaction, the antenna setup geometry and the signal initialization affect the validity of the model, returning a different plasma position. The measurement error is defined as the deviation of the measured position to the real one,

$e(F)=r_{d}(F)-r_{m}(F)$,

where $r_{d}(F)$ is calculated with detected signals and $r_{m}(F)$ is the real position of the cutoff density at the emission line. In order to obtain a reliable measurement, the condition $e(F) \sim 0$ must be satisfied. With the help of the simulations, this quantity can be evaluated with the analysis of the detected synthetic signals. The real position is directly obtained from the input plasma model, which is know a-priori. In DEMO, the maximum absolute error requirement is $1 \mathrm{~cm}$.

\subsection{Plasma model and simulation parameters}

The density data from the EUROfusion official database of the 2017 baseline scenario lacks information in the scrape-off layer (SOL). The density profile $n(\rho)$ was extrapolated in this region by an exponential function with a decay length of $3 \mathrm{~cm}$, compatible to the order of values that is observed in experimental tokamaks $[2,3,25,30]$. An hyperbolic tangent frame was applied at the end of the profile to ensure the density was zero at the wall. The pedestal was connected continuously to the scrape-off layer, as shown in Fig. 1.

Due to the poor resolution of the normalized poloidal flux map $\rho(R$ $Z$ ), the equilibrium density profile $n_{0}(R, Z)$ was calculated extrapolating the normalized poloidal flux to the grid points and using $n_{e}(\rho)$. A region of interest at the high field side of $1 \mathrm{~m} \times 0.6 \mathrm{~m}$ was used in all the simulations of this study. The line of view is aligned perpendicularly to the separatrix, optimizing the detected power and the position error at the equatorial zone (the density profile is approximately slab in the antenna's frame). The relativistic effects were neglected due to the expected low temperatures at the SOL [14]. The plasma is probed in three three different bandwidths, $\mathrm{K}^{\prime}: 18-29 \mathrm{GHz}, \mathrm{Ka}^{\prime}: 29-45 \mathrm{GHz}$ and $\mathrm{Q}^{\prime}$ : 45-54 GHz. They were defined to reduce the number of simulations and do not correspond to any specific designation system. Each band is simulated with 160,000 time steps to save computational time, corresponding to sweeping times in the order of $0.1 \mu$ s. The initialization was done using the WKB phase derivative. There are uncertainties associated with the unobserved low density edge profile, which are not important for the purpose of this study $[10,24,27]$.

\subsection{The density fluctuations modeling}

The total plasma density is the sum of the equilibrium density term $n_{0}$ with the fluctuations term $d n_{e}$,

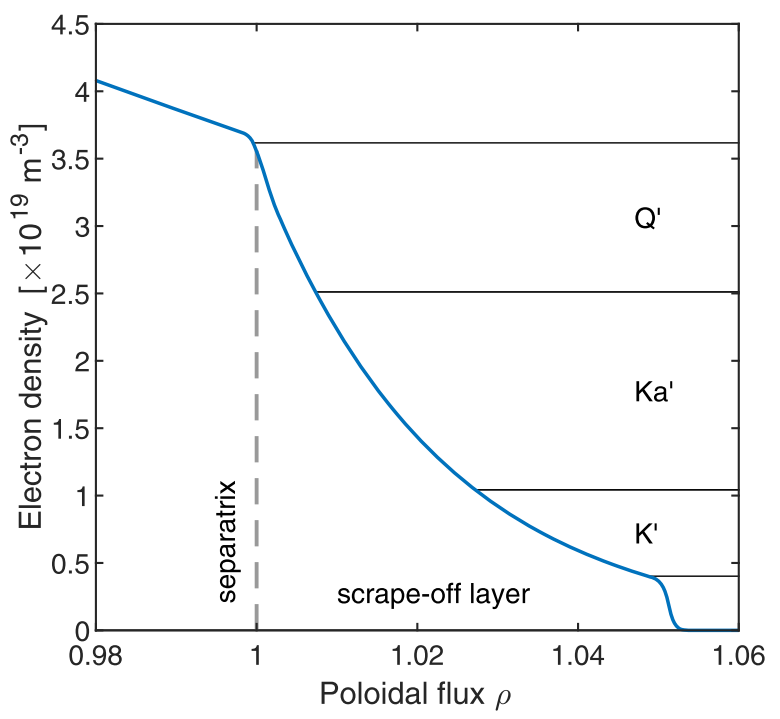

Fig. 1. The extrapolated density profile $n_{e}(\rho)$. 
$n(x, y)=n_{0}(x, y)+d n_{e}(x, y)$

The density fluctuations are locally described by a spectrum and an amplitude/level with respect to the equilibrium plasma. Due to the lack of information on the turbulence properties of DEMO plasmas, we define the fluctuations with an analytical model. We used a Kolmogorov-like spectrum [4,9], following [28]. The spectrum is described by

$S(k)=H\left(k_{\text {knee }}-k\right)+H\left(k-k_{\text {knee }}\right) \times k_{\text {knee }}^{3} k^{-3}$,

where $H(k)$ is the Heaviside function and $k$ is the density fluctuations wavenumber. This model assumes the electron drift waves as the predominant instability at the edge and uses the $k_{\text {knee }} \sim 1 / \rho_{S}$ according to its maximum growth rate [11]. $\rho_{S}$ is the Larmor radius calculated with the sound speed. With the provided values of magnetic field at the separatrix position $(7.2 \mathrm{~T})$ and the considered values for the electron temperature $(3-4 \mathrm{keV}), k_{\text {knee }}$ is approximately $8 \mathrm{~cm}^{-1}$. The aim of this work is the study of the kind of behavior of the measurement error under the variations of the turbulence amplitude. The accuracy of the results can be improved using realistic density profiles from gyrokinetic simulations, as done in Vicente and et al [29]. The fluctuations term is defined as

$d n_{e}(x, y)=n_{0}(x, y) \operatorname{Frm}(x, y) \frac{d n(x, y)}{\operatorname{RMS}[d n(x, y)]}$,

where $d n(x, y)$ is the inverse Fourier transform of the spectrum amplitude $S\left(k_{x}, k_{y}\right)=S\left[k_{x}\right] S\left[k_{y}\right]$ with spectral phase $\phi(x, y)$, and $\operatorname{Frm}(x, y)$ is the frame function. This function localizes the turbulence and defines its amplitude. The turbulence is assumed to be homogeneous and isotropic in the poloidal plane. By defining a random spectrum phase profile $\phi(x$, $y$ ) a different fluctuation matrix $d n(x, y)$ is generated with the Eq. (6). The $d n(x, y)$ matrix is normalized by its RMS (root mean square), so most of the values are in the [1,1] range. Ten levels of turbulence were defined, from $1 \%$ to $10 \%$ in steps of $1 \%$. Five are shown in Fig. 2.

Each frame has a Gaussian shape in the form

$\operatorname{Frm}(\rho)=B+C \exp \left\{-\frac{(\rho-1)^{2}}{2 D^{2}}\right\}$

where $A_{t r b}=B+C$ is the maximum amplitude and $D$ the standard deviation, taken as 0.02 . The $C$ parameter is adjusted in the SOL region and in the pedestal region to produce the required maximum value of amplitude. A hyperbolic tangent frame is applied at the wall position, similarly to the density profile. The turbulence level is low at the core

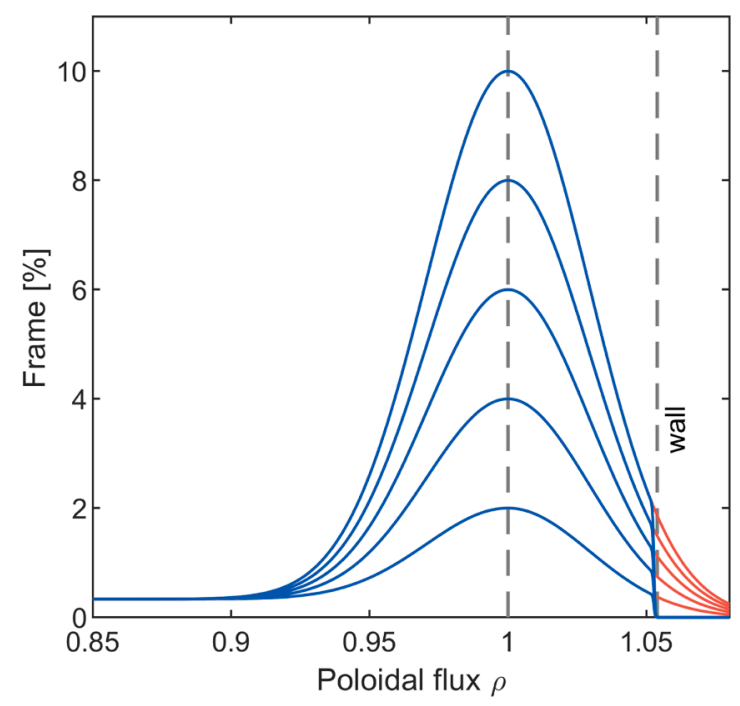

Fig. 2. Five of the ten simulated frames as function of the radial flux coordinate. and increases until the separatrix, where it can reach values above $10 \%$ $[6,16]$. With this model, most of the fluctuations are localized around the separatrix position, contributing with a higher weight to the separatrix error [31]. In the rest of this work, the maximum amplitude $A_{t r b}$ is also referred as level/amplitude of turbulence.

\subsection{The antenna setup and signal detection}

Fig. 3 shows the antenna setup emitting in vacuum by the excitation of the $\mathrm{TE}_{1}$ mode at $40 \mathrm{GHz}$. Only the positive part of the electric field $E_{z}$ is represented in the antenna's frame (propagation parallel to the $\mathrm{x}$ axis). The setup is composed by 6 different elements, a fundamental waveguide, a taper, an oversized waveguide, the antenna, the cavity and the wall.

Thermomechanical studies concluded that a cavity of $10 \mathrm{~cm} \times 10 \mathrm{~cm}$ with rounded corners and the proper cooling system can hold the expected order of wall temperatures. This simple model is used in the setup. An unidirectional source (UTS) is implemented to emit in the plasma direction and detect the reflected signal [22]. Around the FDTD grid there is a Perfectly Matched Layer (PML) that absorbs all the incident energy, so it does not return to the setup when reaches the border. Fig. 4 shows the electric field snapshot as result of probing one turbulent plasma sample with $A_{t r b}=10 \%$ at $53 \mathrm{GHz}$.

When the probing beam is reflected in the plasma, part of its energy enters in the antenna and part is reflected again to the plasma by the wall and the cavity. Part of the energy that is reflected by the wall and the cavity can then be reflected to the structure again, part of the energy disappears due to the PML (at top and bottom) and part of the energy enters in the antenna. As result of this complex plasma-setup interaction and the superposition with the emitting field, the existing electromagnetic field exhibit a complex structure as observed in the electric field snapshot. In the context of the simulations, the propagation between the antenna and the detection system (taper and fundamental waveguide) is reduced to a $10 \mathrm{~cm}$ oversized waveguide. In the real systems the detection is made far away from the antenna. The electromagnetic field is then converted to the $\mathrm{TE}_{1}$ mode using a taper to a fundamental waveguide, where the signal is detected at the central position, $10 \mathrm{~cm}$ before the taper and before the UTS position where only the reflected wave exists. The reason why the detection is done at this distance is because at the beginning of the fundamental waveguide the field may not have a perfect $\mathrm{TE}_{1}$ form due to the propagation of other components of the electromagnetic field, later attenuated. Each simulated frequency band has therefore a different taper and fundamental waveguide. The fundamental mode is excited with a temporal dependence in the form

$S_{s r c}(t)=A_{s r c} \cos [\omega(t) t]$,

where $\omega(t)=K_{f} t . K_{f}=\Delta f / \Delta t$ is the sweeping rate that depends on the frequency band width $\Delta f$ and on the total sweeping time, $\Delta t$. The plasma

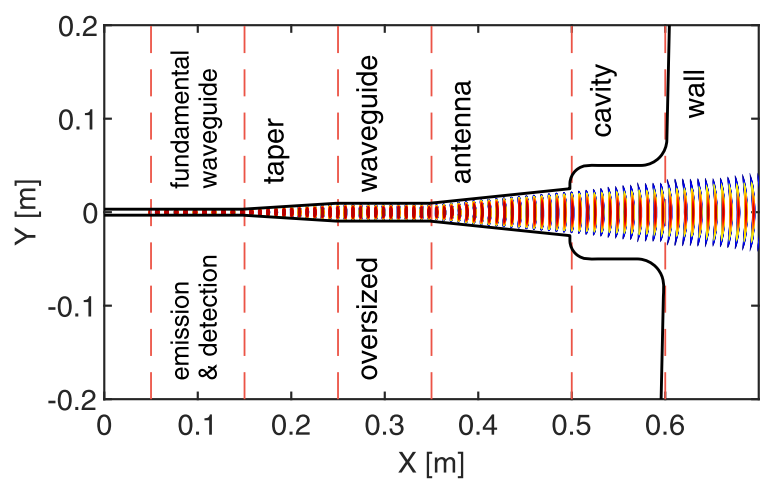

Fig. 3. The antenna setup emitting at $40 \mathrm{GHz}$ with an UTS source emitting unidirectionally a wave in free space. 


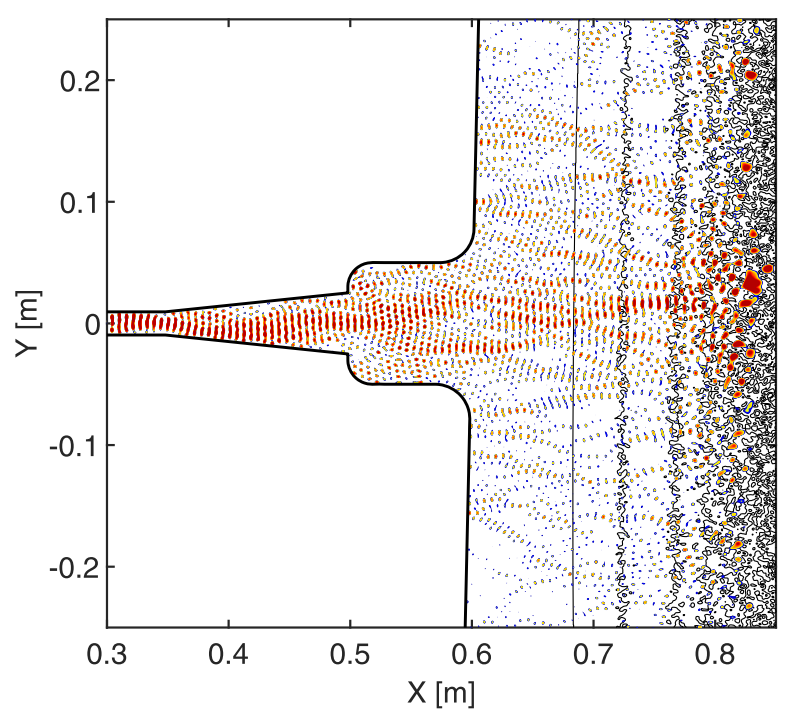

Fig. 4. Electric field snapshot at $53 \mathrm{GHz}$ for $A_{t r b}=10 \%$. The thin black lines correspond to iso-density contours.

is frozen and the stationary state is assumed to be achieved in each probing frequency. In these conditions, the detected signal is written in the form

$S_{\text {det }}(t)=A_{\text {det }}(t) \cos [\omega(t) t+\varphi(t)]$

All the information about the plasma that is possible to extract is in the $\varphi(t)$ and $A_{\text {det }}(t)$ terms. Mixing $S_{\text {src }}(t)$ with $S_{\text {det }}(t)$, a signal with distinct low and high frequency components is obtained:

$S_{m}(t)=\frac{A_{s r c}}{2} A_{d e t}(t)\{\cos [\varphi(t)]+\cos [2 \omega(t) t+\varphi(t)]\}$

By applying a low pass filter, the high frequency component of the signal is removed and the remaining term is proportional to $\cos [\varphi(t)]$. This detection method is known as the homodyne detection. The most common technique to extract the phase derivative from the resulting signal is applying a sliding fast Fourier transform (SFFT) to obtain the beating frequency, which is proportional to the phase derivative. Alternatively, a third signal corresponding to $S_{\text {src }}(t)$ or $S_{\text {det }}(t)$ with a $\pi / 2$ phase shift can be introduced. With the resulting system of equations the phase and the detected amplitude are directly extracted. Defining the $\mathrm{I}(t)$ and $\mathrm{Q}(t)$ signals as

$I(t)=S_{\text {det }}(t) S_{s r c}^{\pi / 2}(t)$,

$Q(t)=S_{d e t}(t) S_{s r c}(t)$,

the phase and the detected amplitude are given by

$\varphi(t)=\arctan \left(\frac{Q(t)}{I(t)}\right)$

$A_{\text {det }}(t)=2 \sqrt{I^{2}(t)+Q^{2}(t)}$.

This is known as the in-phase and quadrature (I/Q) detection scheme (heterodyne system). In the reflectometry simulations the sweeping time is usually much lower than in the experiments to reduce the necessary computational time. This results in a few phase derivative points per band. In this work, we used the I/Q method to calculate the phase derivative that is used to calculate the plasma position. A 4th order low pass Butterworth filter is applied to the I/Q signals to remove the high frequency term and the spurious components (e.g. from the reflections in the wall). The components that affect the zone of characteristic frequencies represent the intrinsic error of the measurement.

\section{The effect of plasma turbulence in the measurements}

Four hundred random generated different plasmas were simulated for each turbulence amplitude. The measurement error $e_{i}$ of every sample $i$ was calculated using the I/Q method and the statistical quantities were calculated [19]. The mean error $E(F)$ and the standard deviation $\sigma_{E}(F)$ of the error were calculated according to the expressions

$E(F)=\frac{1}{N_{s}} \sum_{i=1}^{N_{s}} \mathrm{e}_{i}(F)$

$\sigma_{E}(F)=\sqrt{\frac{1}{N_{s}-1} \sum_{i=1}^{N_{s}}\left[\mathrm{e}_{i}(F)-E(F)\right]^{2}}$,

where $N_{s}$ is the number of considered samples.

The error reliability $E_{R}(F)$ and the rate of failure $E_{F}(F)$ of the measurement are defined by

$E_{R}(F)=\frac{1}{N_{s}} \sum_{i=1}^{N_{s}} H\left[\Delta e(F)-e_{i}\right] H\left[e_{i}(F)+\Delta e\right]$,

$E_{F}(F)=\left[1-E_{R}(F)\right] \times 100(\%)$,

where $\Delta e$ is the error requirement amplitude (the error can be in the range $[-\Delta e, \Delta e]$. Fig. 5 shows the mean error, the standard deviation and the error failure rate at the separatrix as function of the turbulence level.

The respective error distribution for two different turbulence levels is shown on Fig. 6. The first observation is the expected increasing of $\sigma_{E}\left(F_{\text {sep }}\right)$ with the turbulence level. Another observation is the increasing of the absolute error with the turbulence level. The appearance of failed measurements (out of the $1 \mathrm{~cm}$ error requirement) starts around $A_{t r b}=$ $5 \%$, and for the maximum fluctuation level reaches $30 \%$. For the initial levels, the distribution of the error values is centered at the reference error (equilibrium error) with a low standard deviation. As the turbulence level increases, the distribution width increases but the average stills centered at the reference. For turbulence levels greater than $4-$
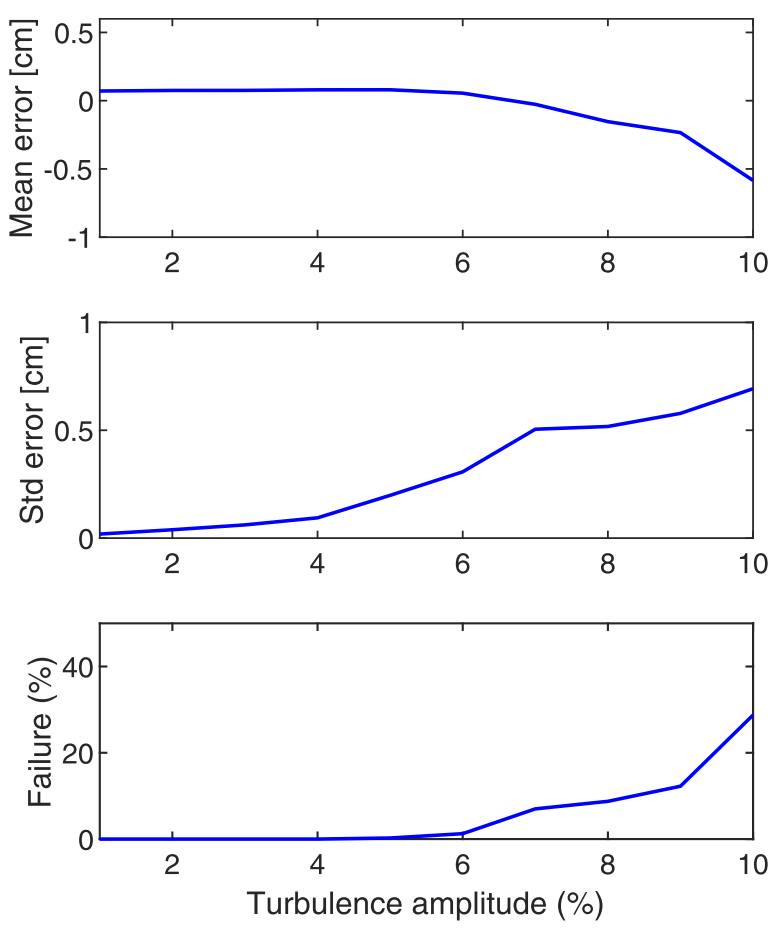

Fig. 5. The mean error at the separatrix $E\left(F_{\text {sep }}\right)$ as function of the turbulence amplitude. 

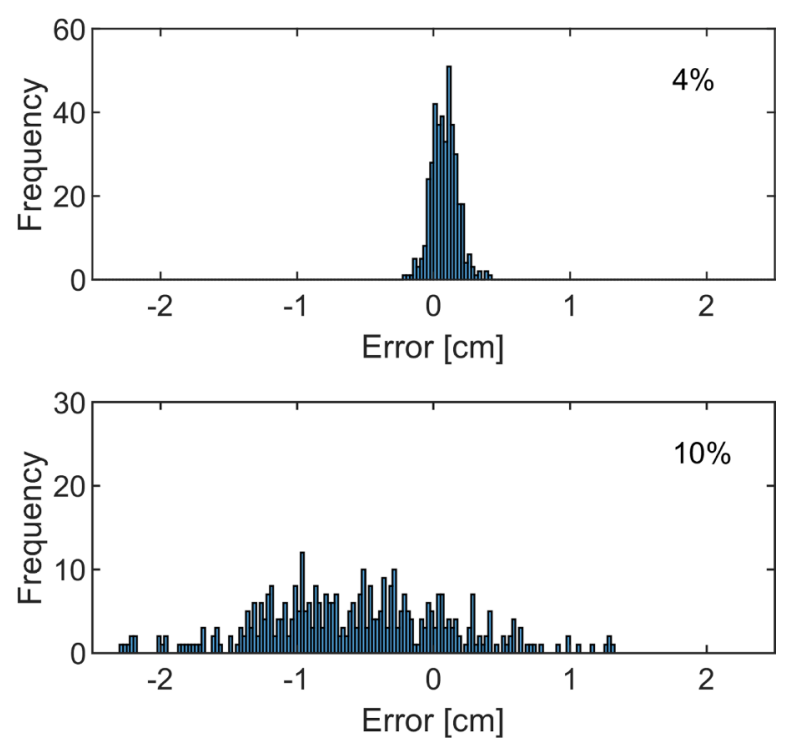

Fig. 6. The error distribution for different turbulence levels.

$5 \%$, the mean becomes negative (measured position closer to the antenna/outward the plasma).

Fig. 7 shows a sample of the phase derivative for a turbulence level of $10 \%$. The phase derivative profiles show oscillations of different amplitudes, depending on the frequency range.

Even though the propagation in a turbulent plasma is a complex phenomena, a one dimensional simple model can explain the tendency to negative errors by the change of the effective cut-off position. First, lets consider a one dimensional density profile in the form

$n(x)=m x+A_{t r b} \cos \left(k_{f} x+\phi_{0}\right)$,

where the first term is a linear density profile with slope $m$ and the second term is an oscillation with amplitude $A_{t r b}$, wavelength $k_{f}$ and phase $\phi_{0}$. By selecting a probing frequency and consequently a cut-off density $n_{c}$, the cut-off position is $x_{c}=n_{c} / m$ if $A_{t r b}=0$ (equilibrium case). By increasing the oscillation amplitude, the new cut-off position $x_{c}^{\prime}$ resulting from the interception of the new profile with the same cut-off density line changes. For low amplitude, it can oscillate around $x_{c}$, depending on $k_{f}$ and $\phi_{0}$. As we increase the amplitude of the oscillation, there is a limit where, for a given $k_{f}$, whatever is the phase there is always a fringe before $x_{c}$ that corresponds to a density higher than the cutoff density. In a plasma with a realistic turbulence profile, this corresponds to the situation in which a great part of the errors become negative as it is observed in the simulations. Since the time delay is

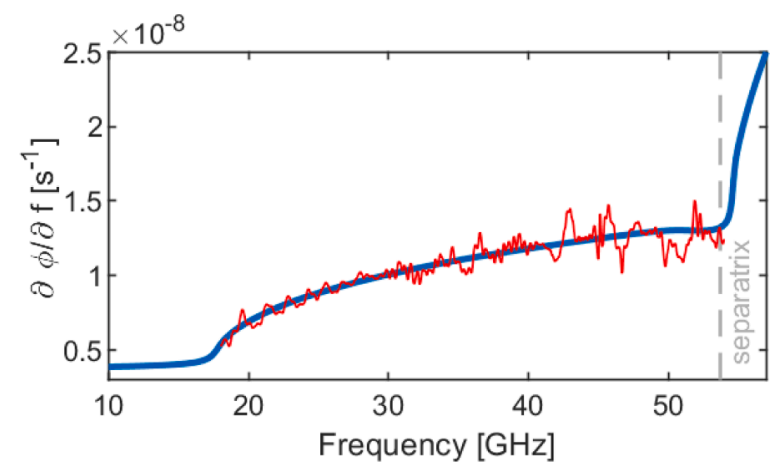

Fig. 7. Phase derivative for two samples with $10 \%$, compared with the WKB theoretical phase derivative (blue). The dashed grey line is the separatrix frequency ( $\sim 53.7 \mathrm{GHz}$ ). (For interpretation of the references to color in this figure legend, the reader is referred to the web version of this article.) decreased due to the reflection occurs before the expected position, there is a tendency on the phase derivative to be below the WKB curve, although in many frequencies it is above or centered. For a fixed frequency, the phase derivative mean is negative.

Using the density profiles from the simulations, we calculated the mean cut-off deviation $\left(x_{c}^{\prime}-x_{c}\right)$ and the respective standard deviation as function of the frequency for different turbulence levels. The line of view centered at the antenna was used as reference. Fig. 8 shows the result.

Similarly to the measurement error, the mean is approximately zero initially and becomes negative for higher turbulence levels. The standard deviation also increases as observed in the measurement error. Since the reflectometry measurements depend on the integral over the frequency range, although with a different weights, this effect is accumulated and increases the final error value. Besides that, the real interaction is much more complex and depend on many other factors, such as the shape and dimension of the turbulent structures, the beam shape or the path before the cut-off.

The effect of the failed measurements in the separatrix reconstruction must be studied to understand what is the range of turbulence that is acceptable naturally by the system and keeps its stability. This range of turbulence can be extended if a position correction in the form

$r^{\prime}(F)=r(F)-E\left(F, A_{t r b}\right)$

is applied. $r^{\prime}(F)$ is the corrected position, $r(F)$ is the obtained position and $E\left(F, A_{t r b}\right)$ is the mean error obtained with the simulations. This correction works if the standard deviation of the error is lower than the measurement requirement, $\sigma_{E}\left(F_{s e p}\right)<\Delta e$, and if the respective number and order of failed measurements is accepted. In a real application, the level of turbulence can be also measured with reflectometry, and this could be adjusted dynamically [8].

\section{Conclusion}

One of the major issues of the DEMO plasma position reflectometer is its reliability due to the plasma turbulence. In this work we studied the position measurements reliability of the DEMO PPR at the HFS using REFMULF, a 2D FDTD code for reflectometry simulations. The turbulence was modulated by a Kolmogorov-like k-spectrum and by a frame function to localize the fluctuations at the separatrix zone as observed in the experiments. Ten different levels of turbulence were defined (1-10\%) and for each one 400 plasmas with the same spectrum and random spectral phase were simulated to obtain the statistical properties of the system. Each signal was analyzed with the I/Q detection method
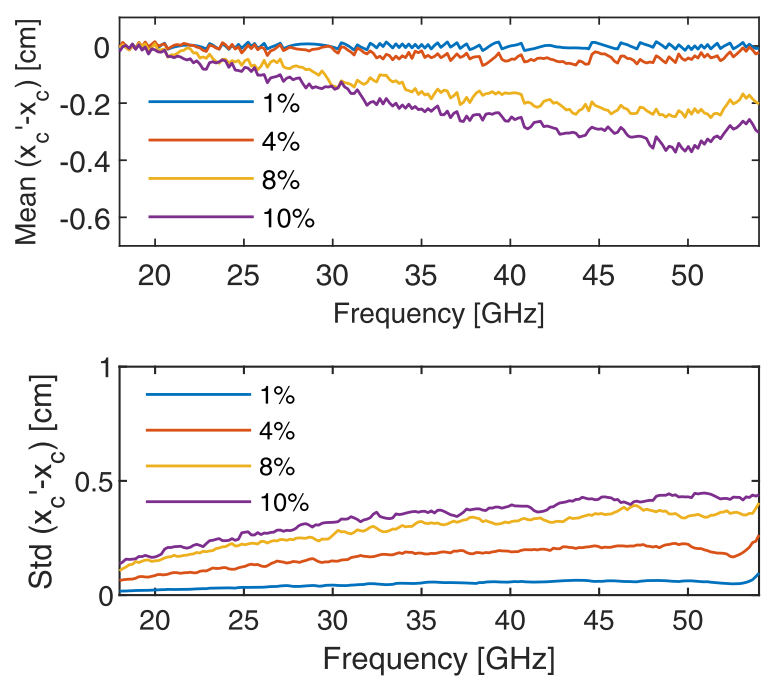

Fig. 8. Mean and standard deviation of the cut-off deviation of the models that were used for simulation. 
and the mean and the standard deviation of the error at the separatrix frequency were calculated for all the plasmas.

The results show that the mean of the measurement error increases in absolute value and becomes negative for higher amplitude of fluctuations. This tendency can be explained by the changes in the effective cutoff position when high level fluctuations occur. This behavior is expected to be dependent on the equilibrium profile and on the local form of the spectrum and amplitude of the turbulence. The standard deviation also increases with the turbulence levels, reaching values in the order of $0.5 \mathrm{~cm}$. At a level of approximately $10 \%$, a considerable number of samples measure the plasma position with an error out of the required condition, $\pm 1 \mathrm{~cm}$. A study on the effect of errors in the order of $1-2 \mathrm{~cm}$ must be done in order to understand if the reconstruction of the separatrix stills possible in these conditions. The range of turbulence accepted by the system can be extended if a correction based on the expected mean error is applied to the position measurements. A system based in reflectometry could measure the turbulence level and correct the position measurements dynamically during the discharge.

The accuracy of the results can be improved including realistic profiles from gyrokinetic simulations or using 3D simulations. In order to prove the reliability of the entire PPR system, it is necessary to apply the same procedure to the other positions of the system (due to different plasma curvature, turbulence spectrum and level), which requires a huge amount of computation time on HPCs to be done. It is also important the study of how the Doppler effect due to the reflection in the propagating structures can affect the position measurements.

\section{Declaration of Competing Interest}

The authors declare that they have no known competing financial interests or personal relationships that could have appeared to influence the work reported in this paper.

\section{Acknowledgments}

This work has been carried out within the framework of the French Federation for Magnetic Fusion Studies (FR-FCM) and EUROfusion Consortium and has received funding from the Euratom research and training programme 2014-2018 under grant agreement no. 633053. The views and opinions expressed herein do not necessarily reflect those of the European Commission. IST activities also received financial support from "Fundação para a Ciência e Tecnologia" through project UID/FIS/ $50010 / 2019$.

\section{References}

[1] W. Biel, et al., Diagnostics for plasma control - from iter to demo, Fusion Eng. Des. 146 (2019) 465-472.

[2] D. Carralero, et al., An experimental investigation of the high density transition of the scrape-off layer transport in ASDEX upgrade, Nucl. Fusion 54 (2014) 123005.

[3] D. Carralero, et al., Recent progress towards a quantitative description of filamentary sol transport, Nucl. Fusion 57 (2017) 056044.
[4] P.H. Diamond, Modern Plasma Physics 1, Cambridge University Press, 2010.

[5] C. Fanack, et al., Ordinary-mode reflectometry: modification of the scattering and cut-off responses due to the shape of localized density fluctuations, Plasma Phys. Control. Fusion 38 (1996) 1915-1930.

[6] T. Gerbaud, et al., Comparison of density fluctuation measurements between omode and x-mode reflectometry on tore supra, Rev. Sci. Instrum. 77 (2006) $10 \mathrm{E} 928$.

[7] E. Gusakov, et al., Strong Bragg backscattering in reflectometry, Plasma Phys. Control. Fusion 51 (2009) 065018.

[8] E. Gusakov, et al., Reconstruction of the turbulence radial profile from reflectometry phase root mean square measurements, Plasma Phys. Control. Fusion 54 (4) (2012) 045008.

[9] T. Happel, et al., Comparison of detailed experimental wavenumber spectra with gyrokinetic simulation aided by two-dimensional full-wave simulations, Plasma Phys. Control. Fusion 59 (2017) 054009.

[10] I.H. Hutchinson, Principles of Plasma Diagnostics, second ed., Cambridge University Press, 2005.

[11] F. Jenko, et al., Global and local gyrokinetic simulations of high-performance discharges in view of ITER, Nucl. Fusion 53 (7) (2013) 073003.

[12] G. Kramer, et al., Antenna-plasma coupling calculations for the iter low-field side reflectometer, Nucl. Fusion 58 (2018).

[13] O. Krutkin. Theoretical Analysis and Full-Wave Simulations Combined in the Development of the Synthetic Doppler Reflectometry Diagnostics for Tokamaks, University of Lorraine, 2020. Ph.D. thesis.

[14] E. Mazzucato, Relativistic effects on microwave reflectometry, Phys. Fluids B 4 (1992) 3460.

[15] E. Mazzucato, et al., Microwave reflectometry for magnetically confined plasmas, Rev. Sci. Instrum. 69 (1998) 2201.

[16] A. Medvedeva, et al., Density profile and turbulence evolution during L-Htransition studied with the ultra-fast swept reflectometer on ASDEX upgrade, Plasma Phys. Controlled Fusion 59 (2017) 125014.

[17] R. Nazikian, et al., A tutorial on the basic principles of microwave reflectometry applied to fluctuation measurements in fusion plasmas, Phys Plasmas 8 (2001) 1840.

[18] E. Ricardo, et al., Assessment of a multi-reflectometers positioning system for demo plasmas, J. Instrum. 14 (2019) C08010.

[19] S. Ross, Introduction to Probability and Statistics for Engineers and Scientists, Student Solutions Manual, fourth ed., Elsevier Science, 2009.

[20] J. Santos, et al., Reflectometry-based plasma position feedback control demonstration at ASDEX upgrade, Nucl. Fusion 52 (2012) 032003.

[21] A. Silva, et al., First assessment of microwave diagnostics for demo, Fusion Eng. Des. 96-97 (2015) 948-951.

[22] F. da Silva, et al., Unidirectional transparent signal injection in finite-difference time-domain electromagnetic codes-Application to reflectometry simulations, J Comput Phys 203 (2005) 467.

[23] F. da Silva, et al., Introducing REFMULF, a 2D full polarization code and REFMUL3, a 3D parallel full wave Maxwell code. Proceedings of the 13th International Reflectometry Workshop, 2017.

[24] A.C.C. Sips, G.J. Kramer, Analysis of reflectometry density profile measurements in jet, Plasma Phys. Controlled Fusion 35 (1993) 743.

[25] P. Stangeby, The Plasma Boundary of Magnetic Fusion Devices, first ed., Institute of Physics Pub, 2000.

[26] P. Varela, et al., Assessment of density profile automatic evaluation from broadband reflectometry data, Rev. Sci. Instrum. 72 (1) (2001) 315.

[27] P. Varela, et al., Initialization of plasma density profiles from reflectometry, Rev. Sci. Instrum. 66 (1995) 4937.

[28] J. Vicente, et al., Turbulence level effects on conventional reflectometry using 2D full-wave simulations, Rev. Sci. Instrum. 89 (2018) 10 H110.

[29] J. Vicente, et al., 2D full-wave simulations of conventional reflectometry using 3D gyro-fluid plasma turbulence, Plasma Phys. Controlled Fusion (2020) 025031.

[30] A. Wynn, et al., Investigation into the formation of the scrape-off layer density shoulder in jet iter-like wall L-mode and H-mode plasmas, Nucl. Fusion 58 (2018) 056001.

[31] G. Zadvitskiy. Experimental and Numerical Study of Turbulence in Fusion Plasmas using Reflectometry Synthetic Diagnostics, Universitde Lorraine, France, 2018. Ph. D. thesis. 